\title{
BMJ Open Cross-sectional observation study to investigate the impact of risk-based stratification on care pathways for patients with chronic kidney disease: protocol paper
}

\author{
Harjeet Kaur Bhachu, ${ }^{1,2}$ Paul Cockwell, ${ }^{2}$ Anuradhaa Subramanian, ${ }^{1}$ \\ Krishnarajah Nirantharakumar, ${ }^{1}$ Derek Kyte, ${ }^{3,4}$ Melanie Calvert ${ }^{5}$
}

To cite: Bhachu HK, Cockwell P, Subramanian A, et al. Crosssectional observation study to investigate the impact of risk-based stratification on care pathways for patients with chronic kidney disease: protocol paper. BMJ Open 2019;9:e027315. doi:10.1136/ bmjopen-2018-027315

- Prepublication history for this paper is available online. To view these files, please visit the journal online (http://dx.doi. org/10.1136/bmjopen-2018027315).

Received 16 0ctober 2018 Revised 19 March 2019 Accepted 2 May 2019

Check for updates

(C) Author(s) (or their employer(s)) 2019. Re-use permitted under CC BY-NC. No commercial re-use. See rights and permissions. Published by BMJ.

For numbered affiliations see end of article.

Correspondence to Dr Paul Cockwell; paul.cockwell@uhb.nhs.uk

\section{ABSTRACT}

Introduction Chronic kidney disease (CKD) management in the UK is usually primary care based, with National Institute for Health and Care Excellence (NICE) guidelines defining criteria for referral to secondary care nephrology services. Estimated glomerular filtration rate (eGFR) is commonly used to guide timing of referrals and preparation of patients approaching renal replacement therapy. However, eGFR lacks sensitivity for progression to end-stage renal failure; as a consequence, the international guideline group, Kidney Disease: Improving Global Outcomes has recommended the use of a risk calculator. The validated Kidney Failure Risk Equation may enable increased precision for the management of patients with CKD; however, there is little evidence to date for the implication of its use in routine clinical practice. This study will aim to determine the impact of the Kidney Failure Risk Equation on the redesignation of patients with CKD in the UK for referral to secondary care, compared with NICE CKD guidance.

Method and analysis This is a cross-sectional population-based observational study using The Health Improvement Network database to identify the impact of risk-based designation for referral into secondary care for patients with CKD in the UK. Adult patients registered in primary care and active in the database within the period 1 January 2016 to 31 March 2017 with confirmed CKD will be analysed. The proportion of patients who meet defined risk thresholds will be cross-referenced with the current NICE guideline recommendations for referral into secondary care along with an evaluation of urinary albumin-creatinine ratio monitoring.

Ethics and dissemination Approval was granted by The Health Improvement Network Scientific Review Committee (Reference number: 18THIN061). Study outcomes will inform national and international guidelines including the next version of the NICE CKD guideline. Dissemination of findings will also be through publication in a peer-reviewed journal, presentation at conferences and inclusion in the core resources of the Think Kidneys programme.

\section{Strengths and limitations of this study}

- The Health Improvement Network is a large UK primary care electronic medical record resource with over 3 million active patients from 787 general practices.

- Due to laboratory variation in measurements, we are standardising estimated glomerular filtration rate (eGFR) using the gold-standard Chronic Kidney Disease Epidemiology Collaboration (CKD-EPI) equation to allow for data comparison; however, some laboratories may still report eGFR using the Modification of Diet in Renal Disease equation.

- The CKD-EPI equation requires knowledge of black ethnicity in order to calculate eGFR; if this information is not available, it could lead to misclassification of patients into stages $3-5$ chronic kidney disease (CKD); individuals of black ethnicity make up 3.3\% of the UK population so the consequence of this may be limited.

- A proportion of people with CKD stages 3-5 will not have been tested for urine albumin-creatinine ratio; this group may include patients who meet the criteria for referral to nephrology services both by the National Institute for Health and Care Excellence (NICE) guideline and/or the Kidney Failure Risk Equation thresholds, therefore reducing the number of the population in the primary analysis.

- The NICE criteria for referral into secondary care that cannot be assessed comprise hypertension that remains poorly controlled despite the use of at least four antihypertensive drugs at therapeutic doses, known or suspected rare or genetic causes of CKD and suspected renal artery stenosis.

\section{INTRODUCTION}

Patients with chronic kidney disease (CKD) are routinely managed in primary care, with National Institute for Health and Care Excellence (NICE) guidelines defining criteria for referral to secondary care nephrology services. ${ }^{1}$ The focus in secondary care is 
primarily on patients with CKD who have progressive or advanced disease and where there is perceived to be a significant risk of progression to end-stage renal failure.

In England, the estimated prevalence of patients with known stage G3a to G5 CKD (estimated glomerular filtration rate $(\mathrm{eGFR})<60 \mathrm{~mL} / \mathrm{min} / 1.73 \mathrm{~m}^{2}$ confirmed on retesting) is $5.2 \% .^{2}$ However, only a small proportion of these patients need to be followed up in secondary care; the large majority are followed up in primary care with a monitoring strategy to identify any decline in eGFR or increase in proteinuria, usually tested by urine albumin-creatinine ratio (ACR). A major consideration for primary care is the timing of referral to secondary care where patients receive ongoing management of CKD progression, treatment for complications of CKD and timely preparation of patients for renal replacement therapy (RRT).

Patients with advanced CKD in secondary care receive information from members of a specialist renal multidisciplinary team regarding end-stage renal failure and the options for RRT or conservative care. In the UK, the number of adult patients initiating RRT in 2015 was 7814 , equivalent to an incidence rate of 120 per million population. ${ }^{3}$ At present, guidelines recommend 'timely referral' for planning RRT, ${ }^{45}$ with patients offered information tailored to their severity of CKD and the risk of progression to allow time for them to fully understand and make informed choices about their treatment. ${ }^{16-9}$

An area of major concern in CKD practice is the lack of precision around the timing of referral to secondary care and preparation for end-stage renal failure. ${ }^{10}$ The trajectory of renal disease progression and therefore the time to end-stage renal failure varies widely between individuals. ${ }^{7-9}$ As a consequence, patients are often uncertain how their disease will progress and have described feeling unprepared for dialysis therapy. ${ }^{11}$

Currently, the level of eGFR is the main parameter used to guide the timing of referral to secondary care nephrology services and preparation for patients approaching advanced CKD and RRT. ${ }^{12}$ The UK Renal Association guidelines recommended that 'most patients whose eGFR is $<30 \mathrm{~mL} / \mathrm{min} / 1.73 \mathrm{~m}^{2}$ and declining should receive timely and personalised information regarding established kidney failure and RRT options so they can make an informed decision about treatment'. ${ }^{6}$ This is also the level of renal function at which NICE recommends specialist referral. ${ }^{1}$ However, most patients with an eGFR $<30 \mathrm{~mL} / \mathrm{min} / 1.73 \mathrm{~m}^{2}$ are likely to be at low risk (defined as $<10 \%)^{13}$ of progressing to RRT in a 2-year period. There is large variation in the risk of progression to RRT as variables that are independent of the eGFR, namely age, gender and urinary ACR are covariates in risk of progression.

The lack of sensitivity of eGFR for progression to RRT was recognised by Kidney Disease: Improving Global Outcomes (KDIGO), which endorsed the use of a validated risk calculator and suggested referral for planning RRT if there is a risk of kidney failure (that may require treatment with dialysis or transplantation) of 10\%-20\% or higher within 1 year. ${ }^{4}$ The Kidney Failure Risk Equation is a generalisable CKD risk prediction model that has been externally validated with patients from over 30 countries worldwide. ${ }^{13}{ }^{14}$ It calculates the 2-year and 5-year probability of progression to end-stage renal failure requiring treatment with dialysis or transplantation for a patient with CKD. Work done by a nephrology group in Canada, defined a risk-based practice where Kidney Failure Risk Equation scores were used to triage referrals into secondary care and to direct patients into the appropriate nephrology clinic and services through the CKD journey. ${ }^{7}$ Furthermore, a threshold of a 5 -year risk to end-stage renal failure of $\geq 3 \%$ for nephrology referral has been integrated and evaluated in a Canadian population; this has shown to benefit a high-risk population by increasing precision in CKD management, including reducing wait times for secondary care review. ${ }^{15}$

Using an eGFR threshold alone, a patient who is at low risk for progression may experience premature planning for treatment for end-stage renal failure, resulting in patient anxiety ${ }^{16}$ and an unnecessary use of resources. ${ }^{17}$ Conversely, patients who are at a higher risk of progression may not be identified early enough to allow sufficient time for education and intervention to enable appropriate dialysis modality decision and access formation or for transplant suitability assessment and listing. For example, using the Kidney Failure Risk Equation, two male patients of 50 years of age with an eGFR of $30 \mathrm{~mL} /$ $\mathrm{min} / 1.73 \mathrm{~m}^{2}$ will have a very different risk of progression to end-stage renal failure when stratified by a urine ACR of $1 \mathrm{mg} / \mathrm{mmol}$ compared with $300 \mathrm{mg} / \mathrm{mmol}$, with respective 2-year and 5-year risks of $1.52 \%$ and $18.22 \%$ and $5.77 \%$ and $54.11 \%$, respectively. ${ }^{6}$

Information provided by the Kidney Failure Risk Equation may help to reduce burden for those at low risk of progression, guide the timing of information for modality planning, improve patient outcomes and offer cost savings to the healthcare system through optimising the use of resources, creating a more efficient pathway for patients with CKD. ${ }^{9} 13$ Other potential benefits include enhancing the accuracy of commissioning and delivery of renal services. Most importantly, it may help patients to understand the path of their chronic illness and in turn allow them to prepare both physically and psychosocially. In the UK, the equation is not widely used in routine clinical care, and there is little known of the benefits to a patient with CKD and the impact on the healthcare system of the incorporation of risk prediction into the clinical pathway. ${ }^{818}{ }^{19}$ Further research to evaluate the impact of prognostic modelling is required..$^{20}$

This study will explore the practical application of risk prediction to end-stage renal failure in patients with CKD by identifying the burden of need in primary care and the impact on renal services in the UK if the Kidney Failure Risk Equation is implemented. This will be addressed by identifying the number of patients who fulfil referral criteria from primary to secondary care nephrology 
using current NICE-based criteria, and these data will be compared with the number ascertained with the Kidney Failure Risk Equation, using risk thresholds for referral into secondary care. Results may also guide adjustments to predefined thresholds for our UK population.

\section{METHODS AND ANALYSIS}

\section{Study design and data source}

This is a cross-sectional population-based observational study using The Health Improvement Network (THIN) database to identify the impact of risk-based designation for patients with CKD in the UK. THIN is a large UK primary care electronic medical record resource with over 3 million active anonymised patients' data. Patients registered in primary care for at least a year and active in the THIN database with an index serum creatinine (SCr) level within the period 1st January 2016 to 31st March 2017 will be included in the study.

The Kidney Failure Risk Equation requires four variables: age, gender, eGFR (calculated from plasma or serum creatinine using the Chronic Kidney Disease Epidemiology Collaboration (CKD-EPI) equation) and urine ACR. While CKD-EPI is the recommended creatinine-based eGFR equation, many laboratories may still be calculating eGFR using the Modification of Diet in Renal Disease (MDRD) equation, and subsequent referrals to secondary care will be based on this result.Serum creatinine ( $\mathrm{SCr}$ ) and ethnicity data, where available, will be used in order to recalculate the eGFR using the CKD-EPI equation.

The variables available in the THIN database allow for integration into the Kidney Failure Risk Equation to calculate the proportion of patients who fulfil criteria for referral into secondary care nephrology services and will be cross-referenced with current NICE guideline referral criteria.

\section{Data collection}

A flow diagram to illustrate the method for data collection is shown in figure 1 . All adults aged 18 years and older will be identified. The first SCr from 1 January 2016 to 31 March 2017 will be collected, and to confirm CKD, a second SCr value will be required $>90$ days from the first. The latest of the two SCr values will be the index SCr. Using age, gender, ethnicity and SCr data, eGFR values will be calculated using the CKD-EPI equation. Those patients meeting the KDIGO definition of CKD, that is, eGFR $<60 \mathrm{~mL} / \mathrm{min} / 1.73 \mathrm{~m}^{2}$ on at least two separate occasions, more than 90 days apart will be identified. All individuals who have a urine ACR level within 12 months from the index SCr will be included in the primary analysis.

With the group of patients identified for the primary analysis, we will establish the impact of redesignation of referrals to nephrology services using the Kidney Failure Risk Equation compared with the current NICE criteria using the eGFR by calculating the number of patients

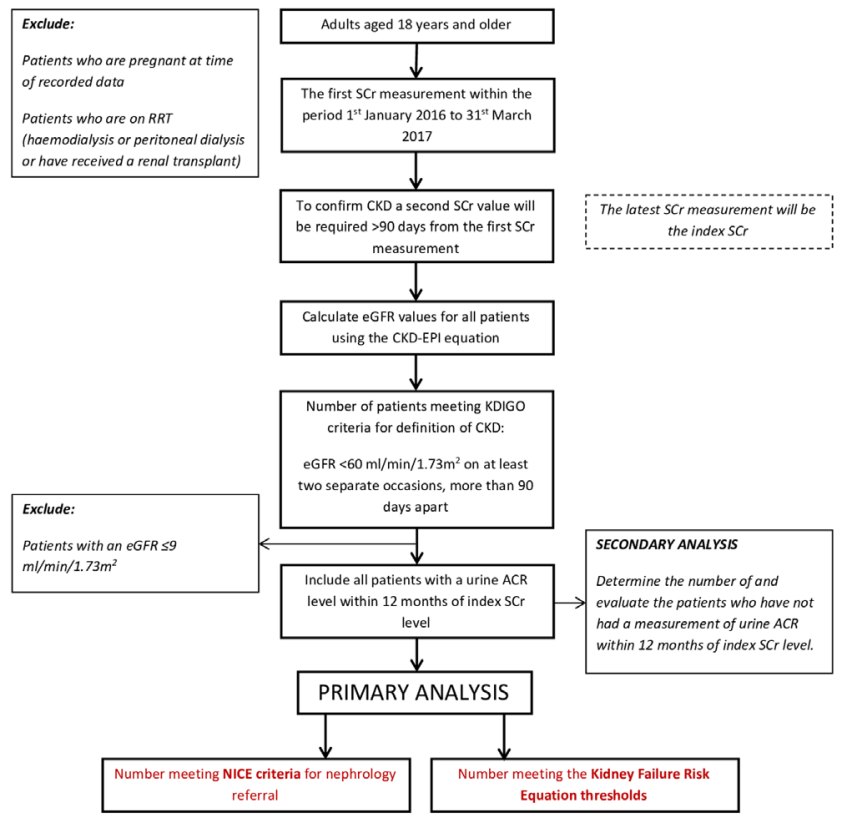

Figure 1 Flow diagram to illustrate the method for data collection. ACR, albumin-creatinine ratio; CKD, chronic kidney disease; CKD-EPI, Chronic Kidney Disease Epidemiology Collaboration; eGFR, estimated glomerular filtration rate; KDIGO, Kidney Disease: Improving Global Outcomes; NICE, National Institute for Health and Care Excellence; RRT, renal replacement therapy.

who meet pragmatic thresholds that are currently in use or being proposed including:

1. NICE criteria of referring with ${ }^{1}$ :

a. An eGFR $<30 \mathrm{~mL} / \mathrm{min} / 1.73 \mathrm{~m}^{2}$.

2. Kidney Failure Risk Equation criteria of:

a. $\geq 3 \%$ risk of end-stage renal failure at 5 years.

b. $\geq 10 \%$ risk of end-stage renal failure at 2 years.

The study will also allow an assessment of the proportion of patients who have available an ACR to allow risk stratification and direct management of CKD. The current NICE-recommended thresholds for ACR based referral into secondary care for a patient with CKD are ${ }^{1}$ :

1. ACR $\geq 70 \mathrm{mg} / \mathrm{mmol}$, unless known to be caused by diabetes and already appropriately treated.

2. ACR $\geq 30 \mathrm{mg} / \mathrm{mmol}$ and haematuria.

For those who have not had their urine ACR measured, the secondary analysis will establish the number of patients who, according to the current NICE CKD guidance, should have their urine ACR measured annually if they have an eGFR $<60 \mathrm{~mL} / \mathrm{min} / 1.73 \mathrm{~m}^{2}$, that is, have CKD. ${ }^{1}$ This group may include patients who meet criteria for referral to nephrology services by eGFR and so absence of ACR recording will be investigated. Baseline characteristics data and severity of CKD in this group will be reviewed and compared with those with their urine ACR recorded. Baseline characteristics chosen are consistent with NICE CKD guidance, which recommends offering testing for CKD using eGFR and/or ACR in people with risk factors for $\mathrm{CKD}{ }^{1}$ 
Study inclusion criteria:

- Adults aged 18 years and older.

- Must have two recorded SCr values at least 90 days apart.

Study exclusion criteria:

- Patients who are pregnant at time of recorded data.

- Patients who have reached end-stage renal failure:

- On RRT (haemodialysis, peritoneal dialysis or have received a renal transplant).

- $\quad$ eGFR $\leq 9 \mathrm{~mL} / \mathrm{min} / 1.73 \mathrm{~m}^{2}$

- Patients with eGFR $\geq 60 \mathrm{~mL} / \mathrm{min} / 1.73 \mathrm{~m}^{2}$.

\section{Study variables}

Using age (Years), gender, ethnicity (black or Other) and the index serum creatinine values $(\mu \mathrm{mol} / \mathrm{L})$, eGFR $(\mathrm{mL} /$ $\mathrm{min} / 1.73 \mathrm{~m}^{2}$ ) will be calculated using the CKD-EPI equation. The Kidney Failure Risk Equation requires four variables: age (years), gender, eGFR $\left(\mathrm{mL} / \mathrm{min} / 1.73 \mathrm{~m}^{2}\right)$ and urine ACR $(\mathrm{mg} / \mathrm{mmol})$.

Read codes, a set of clinical terms that describe the medical history of patients in the THIN database, will be used to identify the comorbidity data and allow described patients to be excluded from the analysis. Patients who reach end-stage renal failure are defined as those who have commenced RRT or follow a conservative care (nonRRT) pathway. Read codes will identify those who are pregnant or are receiving RRT (dialysis or renal transplant) at time of data collection enabling their exclusion from the analysis. No clearly defined Read code is available for patients on a conservative care pathway so patients with an eGFR $\leq 9 \mathrm{~mL} / \mathrm{min} / 1.73 \mathrm{~m}^{2}$, the mean eGFR at which a patient with CKD commences RRT in the $\mathrm{UK}^{3}{ }^{3}$ will be excluded from the study.

The following baseline characteristics will be collected: diabetes, hypertension, acute kidney injury, ischaemic heart disease, chronic heart failure, peripheral vascular disease, cerebrovascular disease, calcineurin inhibitor (tacrolimus and ciclosporin)/lithium use, renal calculi/ prostatic disease, systemic lupus erythematosus and haematuria, plus haemoglobin and serum phosphate, both measured within 12 months of the $\mathrm{SCr}^{1}{ }^{121}$

NICE CKD recommendation advises at least annual quantification of urinary albumin or protein loss for people with an eGFR $<60 \mathrm{~mL} / \mathrm{min} / 1.73 \mathrm{~m}^{2}{ }^{1}$ Evaluation of patients with CKD who have not had an ACR measurement within 12 months of the index SCr will identify patients who have had their ACR tested greater than 1 year from the SCr value or have had alternative measurements such as urinalysis, protein:creatinine ratio or 24 hours urine protein excretion, investigated using available Read codes, within 12 months from the index Scr. The proportion of patients who have not been tested for urinary albumin or protein loss will be calculated.

\section{Analysis}

STATA V.14.2 will be used for data collection and analysis. The number of patients who meet the criteria for

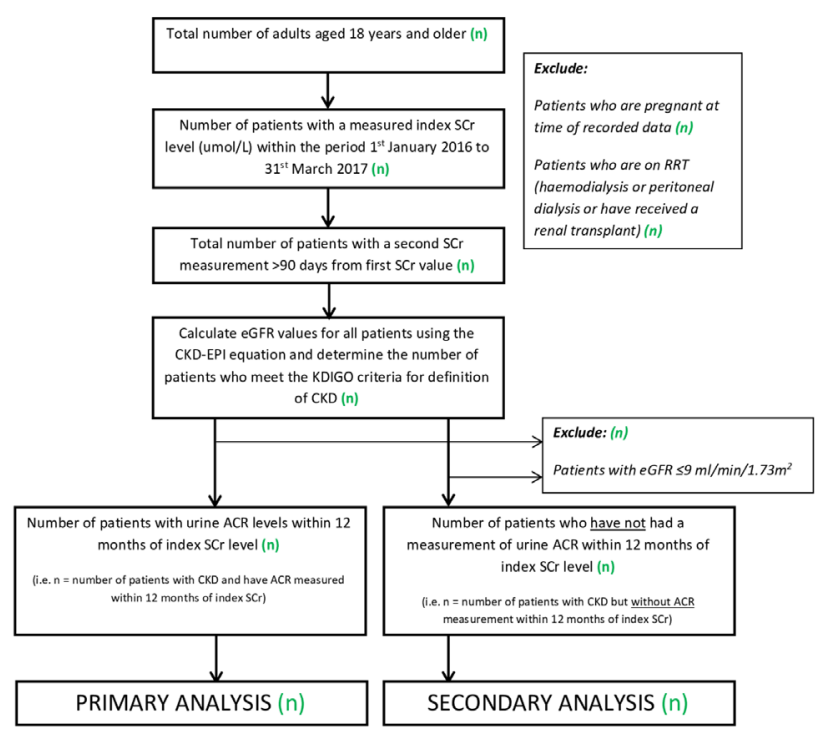

Figure 2 Study population. ACR, albumin-creatinine ratio; CKD, chronic kidney disease; CKD-EPI, Chronic Kidney Disease Epidemiology Collaboration; eGFR, estimated glomerular filtration rate; KDIGO, Kidney Disease: Improving Global Outcomes; RRT, renal replacement therapy.

inclusion into the primary and secondary analyses will be identified (see figure 2).

\section{Primary analysis}

The proportion of patients meeting the current NICE criteria for referral to secondary care nephrology services will be compared with the proportion meeting defined Kidney Failure Risk Equation thresholds. A net reclassification approach will be applied to determine the patients reclassified according to risk thresholds. We will estimate at which risk threshold equipoise is reached compared with current NICE guidelines for referral.

\section{Secondary analysis}

The baseline characteristics of those with CKD stage 3-5 (predialysis) will be described with categorical variables as $\mathrm{n}(\%)$ and numerical data as median (IQR). The differences will be observed between patients who do and do not have a urine ACR documented within 12 months of index SCr. Patients without a urine ACR measurement within 12 months of the index SCr will be evaluated to identify the number and proportion who have had alternative measurements of urinary protein loss, measurements of ACR or alternative outside of the specified study time criteria and those who have not been checked for urinary protein loss.

\section{Patient and public involvement}

This study was informed by the Kidney Health Delivering Excellence strategy, led by Kidney Care UK, the major UK kidney patient charity, which included all relevant UK patient-including organisations and developed a strategy based on patient and professional dialogue. Included within the strategy are the recommendations: '(1) 
Awareness.of the factors that increased the risk of kidney disease...'. This recommendation will be addressed by increasing the use of ACR in referral pathways and using ACR in risk stratification; '(6) Preparation and Choice... patients approaching end-stage renal disease... are given sufficient time and support to prepare for a treatment'. This will be addressed by using an approach that identifies patients with precision for risk to end-stage renal disease. In addition to addressing these recommendations, the study addresses recommendations 15 and 16 , which specifically deal with research and quality improvement, respectively. ${ }^{22}$

\section{Limitations}

Data recorded in the THIN database for eGFR values may be calculated locally using the MDRD or CKD-EPI equations depending on the laboratory. Both NICE guidelines and Kidney Failure Risk Equation recommend eGFR values using the CKD-EPI creatinine equation as it has been shown to more accurately estimate the eGFR and classify fewer patients with CKD ${ }^{23}$ However, many referrals to secondary care are still based on eGFR laboratory values that use the MDRD equation. Therefore, in this study, we will collect data to enable calculation of eGFR using the CKD-EPI creatinine equation. There may be incomplete data due to variables not being recorded. Most importantly, ethnicity, in particular black ethnicity, is a variable used to calculate the eGFR using the CKD-EPI equation. Without this being fully recorded, there may be an underestimate of renal function for some patients who are of black race but have these data missing, potentially leading to an overestimate of patients in stages 3-5 CKD. Individuals of black ethnicity make up $3.3 \%$ of the UK population $^{24}$ so the consequence of this may be small.

The confines of the data collection from THIN database mean it will be challenging to accurately assess CKD progression as defined by the NICE guidelines. There will be variation in the timing and number of SCr measurements a patient may have. NICE CKD guidelines recommend a minimum of three eGFR values of a period of not less than 90 days, and in people with a new finding of a reduced eGFR, it should be repeated within 2 weeks to exclude acute decline. ${ }^{1}$

In patients with CKD and an ACR of $70 \mathrm{mg} / \mathrm{mmol}$ or more, it may be difficult to identify with certainty if it is caused by diabetes and then to assess if the diabetes is appropriately treated without review of the individual patient. This limitation will be addressed by reporting the number of patients with an ACR of $70 \mathrm{mg} / \mathrm{mmol}$ or more and diabetes but not subdividing the group between patients who may or may not have diabetes causing the CKD.

The NICE criteria define that patients with CKD should be referred for hypertension that remains poorly controlled despite the use of at least four antihypertensive drugs at therapeutic doses, known or suspected rare or genetic causes of CKD or suspected renal artery stenosis. In mainstream clinical renal practice, the numbers of these patients are likely to be low, and patients currently being referred cannot be estimated accurately. THIN does not allow for an assessment of this.

NICE CKD recommendations advise at least annual quantification of urine albumin or protein loss for people with an eGFR $<60 \mathrm{~mL} / \mathrm{min} / 1.73 \mathrm{~m}^{2}$ with or without diabetes and patients with an eGFR of $\geq 60 \mathrm{~mL} /$ $\min / 1.73 \mathrm{~m}^{2}$ if there is a strong suspicion of CKD. ${ }^{1}$ The National CKD Audit showed there may be a number of people who have not been tested for urine ACR with stage 3-5 CKD and some general practitioners only send urine for ACR testing if the urine dipstick test is positive. ${ }^{21}$ Absence of ACR data in patients with CKD as a confounding factor will be investigated in this study by identifying if they have any ACR measurements outside of the specified time criteria or alternative measurements of albumin or protein loss such as urinalysis, protein:creatinine ratio or 24 hours urine protein excretion within 12 months of the index SCr. The latter group may include patients who meet criteria for referral to nephrology services by eGFR and may reveal extra potential patients who could have been included in the primary analysis if urine ACR was the unit measured instead.

The Kidney Failure Risk Equation uses single-timepoint variables to calculate risk and does not take into account previous values or changing laboratory test results over time. ${ }^{25}$ A dynamic model compared with a static model (eight-variable Kidney Failure Risk Equation) only modestly improves the powerful risk prediction of the Kidney Failure Risk Equation, ${ }^{25}$ as demonstrated by the C statistic: 0.91 (95\% CI 0.89 to 0.93 ) versus 0.90 (95\% CI 0.88 to 0.92$)$.

\section{DISCUSSION}

This study will explore the practical application of risk prediction for end-stage renal failure in patients with CKD by identifying the burden of need in primary care and the impact on renal services in the UK if the Kidney Failure Risk Equation is implemented. At present, the difference in number of referrals in our UK population and the potential redesignation of patients using a riskbased model is unclear.

To our knowledge, there are no studies on a population-based level to assess the impact of criteria for referral on clinical outcomes. The current NICE guidelines do not map on to long-term clinical outcomes; however, the Kidney Failure Risk Equation does; requiring RRT is a major clinical end-point associated with high mortality and other adverse clinical, patient reported and health economic outcomes.

However, to model the impact of the intervention would require a step-wedge study in enrolling geographical areas with correction for multiple confounders. Assessing the current NICE criteria against outcomes is challenging in that it would not correct for confounding by factors that are common in patients with advanced CKD including frailty. However, the Kidney Failure Risk Equation is validated 
and includes death as a competing risk of progression to end-stage renal failure. Ultimately, a study comparing the impact of an intervention (referral) against criteria that have not been mapped against outcome, with criteria that have been mapped against outcome may not be achievable. In resource-limited healthcare systems (all healthcare systems), a starting point is defining the impact at a population level of a transition from one system that is opinion based (current) to a system that uses a tool that has a quantifiable risk of the end-point, which is the rationale for follow-up in specialist nephrology services; this study is designed to do that.

The outcome of this study may direct a potential change in CKD management pathways, increasing the precision with which patients are identified for referral into secondary care nephrology services. As a consequence, implementing this change will require consideration of incorporation of the Kidney Failure Risk Equation into clinical informatics systems that will enable close working to define appropriate models for delivery of renal services, prompt identification of individuals with increased risk of progression and produce an increased focus on identification of core covariables in particular ACR and ethnicity, which are inadequately checked and recorded. ${ }^{21}$

The health economic impact will need to be explored, from requirements for implementing change to cost savings with improved efficiency. Delivering a risk-based service will require consideration of how patients with CKD interpret risk counselling, the optimal method of communication of risk, preferences on how and when information is shared and training of healthcare professionals.

Further research based on the outcome of this study will be necessary to establish and assess risk-based renal referral and management pathways, investigate appropriate incorporation and communication of risk to patients, education and training needs of healthcare professionals and the health economic impact of integration of the Kidney Failure Risk Equation into clinical practice.

\section{ETHICS AND DISSEMINATION}

The outcome of the results to individually risk stratify patients with CKD may provide evidence towards a change in renal referral pathways. The use of the Kidney Failure Risk Equation may identify a more efficient and possibly more economical service model. The study outcomes will inform national and international guidelines including the next version of the NICE CKD guideline. Dissemination of findings will also be through publication in a peer-reviewed journal and presentation at conferences.

\section{Study status}

Ethics permission has been granted and the data obtained for the study. The study is in data checking and analysis programming phase, and study finish is scheduled for August 2019.
Author affiliations

${ }^{1}$ Institute of Applied Health Research, University of Birmingham, Birmingham, UK ${ }^{2}$ Department of Nephrology, University Hospitals Birmingham NHS Foundation Trust, Queen Elizabeth Hospital Birmingham, Birmingham, UK

${ }^{3}$ Centre for Patient Reported Outcomes Research, Institute of Applied Health Research, University of Birmingham, Birmingham, UK

${ }^{4}$ NIHR Biomedical Research Centre (BRC) in Inflammatory Diseases, University of Birmingham, Birmingham, UK

${ }^{5}$ Centre for Patient Reported Outcomes Research and Institute of Applied Health Research, University of Birmingham, Birmingham, UK

Contributors HKB, PC, MC and DK conceived the work. HKB, PC, AS and KN designed the protocol. HKB and PC drafted the manuscript; all authors revised it critically for important intellectual content and agree to be accountable for accuracy and integrity of the work.

Funding The authors have not declared a specific grant for this research from any funding agency in the public, commercial or not-for-profit sectors.

Competing interests $\mathrm{HKB}$ and $\mathrm{PC}$ have nothing to disclose. MC reports personal fees from Takeda, personal fees from Ferring, personal fees from Astellas, outside the submitted work. KN reports grants from National Institute for Health Research, grants from MRC, grants from Diabetes UK, grants from AstraZeneca, personal fees from MSD, personal fees from Sanofi, personal fees from BI, outside the submitted work. AS has nothing to disclose. DK reports grants from NIHR and Macmillan Cancer Support and personal fees from Merck.

Patient consent for publication Not required.

Ethics approval Approval for this study has been granted by The Health Improvement Network Scientific Review Committee (reference number: 18THIN061).

Provenance and peer review Not commissioned; externally peer reviewed.

Open access This is an open access article distributed in accordance with the Creative Commons Attribution Non Commercial (CC BY-NC 4.0) license, which permits others to distribute, remix, adapt, build upon this work non-commercially, and license their derivative works on different terms, provided the original work is properly cited, appropriate credit is given, any changes made indicated, and the use is non-commercial. See: http://creativecommons.org/licenses/by-nc/4.0/.

\section{REFERENCES}

1. National Institute for Health and Care Excellence. Chronic kidney disease in adults: assessment and management (CG182. London, UK: NICE, 2014

2. Fraser SD, Aitken G, Taal MW, et al. Exploration of chronic kidney disease prevalence estimates using new measures of kidney function in the health survey for England. PLoS One 2015;10:e0118676.

3. Gilg J, Methven S, Casula A, et al. UK Renal Registry 19th Annual Report: Chapter 1 UK RRT Adult Incidence in 2015: National and Centre-specific Analyses. Nephron 2017;137(Suppl 1):11-44.

4. Kidney Disease: Improving Global Outcomes (KDIGO) CKD Work Group, KDIGO 2012 Clinical Practice Guideline for the Evaluation and Management of Chronic Kidney Disease. Kidney Inter suppl 2013:3:1-150.

5. Farrington K, Warwick G. Renal Association Clinical Practice Guideline on planning, initiating and withdrawal of renal replacement therapy. Nephron Clin Pract 2011;118:c189-208.

6. The Kidney Failure Risk Equation. http://kidneyfailurerisk.com/ (Cited 29th Sep 2016).

7. Wojciechowski P, Tangri N, Rigatto C, et al. Risk Prediction in CKD: The Rational Alignment of Health Care Resources in CKD 4/5 Care. Adv Chronic Kidney Dis 2016;23:227-30.

8. Green JA, Boulware LE. Patient Education and Support During CKD Transitions: when the possible becomes probable. Adv Chronic Kidney Dis 2016;23:231-9.

9. Levin A, Djurdjev O, Beaulieu M, et al. Variability and risk factors for kidney disease progression and death following attainment of stage 4 CKD in a referred cohort. Am J Kidney Dis 2008;52:661-71.

10. Treit K, Lam D, O'Hare AM. Timing of dialysis initiation in the geriatric population: toward a patient-centered approach. Semin Dial 2013;26:682-9.

11. Schell JO, Patel UD, Steinhauser KE, et al. Discussions of the kidney disease trajectory by elderly patients and nephrologists: a qualitative study. Am J Kidney Dis 2012;59:495-503. 
12. O'Hare AM, Batten A, Burrows NR, et al. Trajectories of kidney function decline in the 2 years before initiation of long-term dialysis. Am J Kidney Dis 2012;59:513-22.

13. Tangri N, Stevens LA, Griffith J, et al. A predictive model for progression of chronic kidney disease to kidney failure. JAMA 2011;305:1553-9.

14. Tangri N, Grams ME, Levey AS, et al. Multinational Assessment of Accuracy of Equations for predicting risk of kidney failure: a metaanalysis. JAMA 2016;315:164-74.

15. Hingwala J, Wojciechowski P, Hiebert B, et al. Risk-Based triage for nephrology referrals using the kidney failure risk equation. Can $J$ Kidney Health Dis 2017;4:1-9.

16. Daker-White G, Rogers A, Kennedy A, et al. Non-disclosure of chronic kidney disease in primary care and the limits of instrumental rationality in chronic illness self-management. Soc Sci Med 2015;131(Supplement C):31-9.

17. Grams ME, Coresh J. Predicting Risk of RRT in Patients with CKD. Clin J Am Soc Nephrol 2017;12:3-4.

18. Chiu HH, Tangri N, Djurdjev O, et al. Perceptions of prognostic risks in chronic kidney disease: a national survey. Can J Kidney Health Dis 2015:2:88.

19. Echouffo-Tcheugui JB, Kengne AP. Risk models to predict chronic kidney disease and its progression: a systematic review. PLoS Med 2012;9:e1001344.
20. Eckardt KU, Bansal N, Coresh J, et al. Improving the prognosis of patients with severely decreased glomerular filtration rate (CKD G4+): conclusions from a Kidney Disease: Improving Global Outcomes (KDIGO) Controversies Conference. Kidney Int 2018;93:1281-92.

21. Nitsch DCB, Hull S, Wheeler DC. National Chronic Kidney Disease Audit. National Report (Part 1). London, UK: London School of Hygiene \& Tropical Medicine, 2017.

22. Kidney Health Progress Report 2017. 2019 https://www.google. $\mathrm{com} /$ url? sa $=\mathrm{t} \& \mathrm{rct}=\mathrm{j} \& \mathrm{q}=\&$ esrc $=\mathrm{s} \&$ source $=$ web\&cd $=1 \& \mathrm{cad}=\mathrm{rja} \&$ uact $=8 \&$ ved $=2$ ahUKEwj384yn9OfgAhX_RBUIHW6fD9QQFjAA egQIBRAC\&url=https\%3A\%2F\%2Fwww.kidneycareuk.org\% 2Fdocuments\%2F51\%2FKidney_Health_progress_report_2017. pdf\&usg=AOvVaw3mw-4iY3c9tjE7tjjpV17I (Cited 14th Mar 2019).

23. Matsushita K, Mahmoodi BK, Woodward M, et al. Comparison of risk prediction using the CKD-EPI equation and the MDRD study equation for estimated glomerular filtration rate. JAMA 2012;307:1941-51.

24. Ethnicity and National Identity in England and Wales 2011, Office of National Statistics, Editor. 2012

25. Tangri N, Inker LA, Hiebert B, et al. A Dynamic predictive model for progression of CKD. Am J Kidney Dis 2017;69:514-20. 